\title{
Fast and curious
}

\author{
Rémy Pawlak and Tobias Meier discuss how they got involved in the first international NanoCar Race and \\ their impressions from this experience.
}

$\mathrm{T}$ he first time we heard about the NanoCar Race was when Christian Joachim, director of research at the CNRS in France and organizer of the race, was promoting the event during an invited talk at a conference. Enthusiastic as usual, he was explaining in front of a stomached scientific audience his project about a race between 'molecular machines' propelled with electrons from scanning tunnelling microscopes on a gold surface. The project was already at an advanced stage and with several applicants from strong international research groups. We were astounded, but also tickled by the idea: we were curious to know how far, and how fast, we could make a molecule go.

Confident about our expertise in using scanning tunnelling microscopy and atomic force microscopy at low temperature to mechanically manipulate single molecules, we decided to enter the race. But here, the aim was to propel a molecule only using electrons over a hundred nanometres without mechanical pushing. We needed to build up a robust and electrically powered vehicle. This was uncharted territory for us.

The inspiration for the Swiss Nano Dragster, our nanocar, came from our research on single-molecule tribology the study of friction at the molecular level. To choose the appropriate molecule, we turned the problem upside-down and focused on the racetrack. We knew that what determines a fast nanocar is mainly friction and adhesion. But, how could we reduce the friction between the molecule and the gold while maintaining control

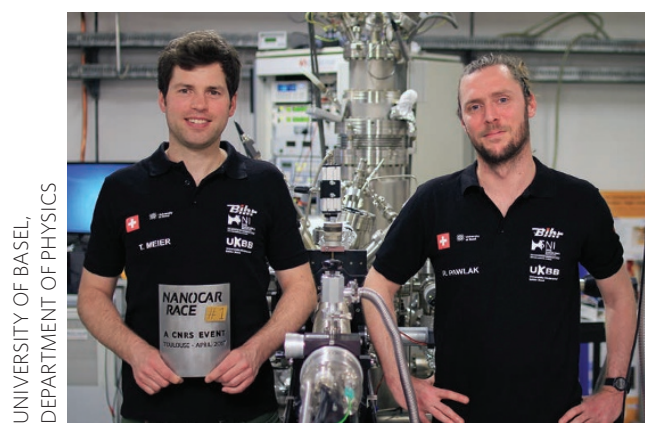

over the motion? From our expertise, we also knew that individual molecules would be easier to work with than large molecular assemblies. The molecule should also survive thermal evaporation in ultra-high vacuum.

With the help of Professor

Catherine Housecroft at the

University of Basel, we selected the $4^{\prime}$-(4-tolyl)-2,2':6',2"'-terpyridine molecule, which we had already used as a sensitizer component in a solar cell project. We could immediately envision that such an aromatic molecule would lie flat on the surface. At the rear, the terpyridine moiety would locally enhance the molecule adsorption on gold; whereas at the front, the tolyl unit would weakly interact with gold and act as a front spoiler, balancing the friction forces during motion. However, only experimental test runs could confirm these predictions. At that point, motorization was still an open question.

The first scanning tunnelling microscopy experiments in Basel revealed molecules individually adsorbed all over the gold surface like snowflakes on a table. Clumsily, we tried to excite one and, to our delight, it moved! Half a nanometre. A few pulses later, the molecule had disappeared from the surface and got attached to the tip apex. A run of only a few nanometres made of short jumps would not be good enough for the NanoCar Race.

Sincerely, at that time, we had no control over the molecule motion. We thus began an iterative series of experiments to disentangle molecule adsorptions, excitations and diffusion. We found that uncontrolled translations are induced by exciting molecular vibrations through the injection of tunnelling electrons into the molecular orbitals. The breakthrough came when we discovered that the voltage applied to the tip creates an electric field that dictates the direction of motion. Luckily, a negative charge density is located at the terpyridine due to a charge transfer with the surface, making the molecule sensitive to the electric field gradient. Depending on the field polarity, the molecule is either attracted towards or repelled from the tip.
This was the clue that motorized our dragster. We started to 'drive' over a few tens of nanometres, exploring our playground with a speed of $20 \mathrm{~nm}$ per hour! But new issues immediately appeared. These were related to the inhomogeneity of the $\mathrm{Au}(111)$ herringbone reconstruction, leading to drastic variation of the molecule diffusion and numerous surface pitfalls. We struggled for days but in the end we trained ourselves on how to slalom between obstacles on the surface. Much like pilots in a rally raid, we reported all traps and dangers experienced by our nanocar in a road book.

The next step was to test the Nano Dragster in Toulouse with the four-probe microscope to be used for the actual race. We had to drive in a different cockpit with a different handling, but using the same propelling mechanisms. Without experience with this machine, we had to prove our pilot skills. We received lots of support from the local researchers and the pilots of other teams as well. We realized that beyond the competition the event was also a fantastic scientific collaboration and its success also depended on all of us solving the common experimental problems inherent to the race conditions.

Finally, the day of the race arrived. We completed our $133 \mathrm{~nm}$ gold racetrack in 6 hours and 30 minutes. Practically in the blink of an eye! We ranked first, exhausted but delighted to simply cross the finish line. The NanoCar Race was an exciting moment to share live on YouTube, showing how scientific experiments are done and how scientists fight for their results. Certainly, all nanocars at the race were in their infancy and lots of improvements are possible. Only close collaborations between chemists and physicists will bring new design concepts and novel propulsion modes.

\section{RÉMY PAWLAK and TOBIAS MEIER are postdoctoral fellow and $\mathrm{PhD}$ student, respectively, in the research group of Professor Ernst Meyer at the University of Basel, Department of Physics, Klingelbergstr. 82, 4056, Basel, Switzerland. e-mail:remy.pawlak@unibas.ch; tobias.meier@unibas.ch}

